\title{
Neutrophil to lymphocyte ratio and adverse clinical outcomes in patients with ischemic stroke
}

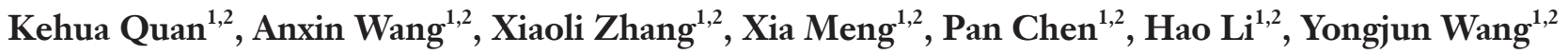 \\ ${ }^{1}$ Department of Neurology, Beijing Tiantan Hospital, Capital Medical University, Beijing, China; ${ }^{2}$ China National Clinical Research Center for \\ Neurological Diseases, Beijing Tiantan Hospital, Capital Medical University, Beijing, China \\ Contributions: (I) Conception and design: K Quan, Y Wang; (II) Administrative support: X Meng, P Chen, H Li, Y Wang; (III) Provision of study \\ materials or patients: A Wang, X Meng, P Chen, H Li, Y Wang; (IV) Collection and assembly of data: K Quan, X Zhang; (V) Data analysis and \\ interpretation: K Quan, A Wang, X Zhang; (VI) Manuscript writing: All authors; (VII) Final approval of manuscript: All authors. \\ Correspondence to: Yongjun Wang. Department of Neurology, Beijing Tiantan Hospital, Capital Medical University, No. 119 South 4th Ring West \\ Road, Fengtai District, Beijing 100070, China. Email: yongjunwang@ncrend.org.cn.
}

Background: Neutrophils and lymphocytes mediate differential inflammatory responses after ischemic stroke and have different effects on patients' clinical outcomes. Several studies have used the neutrophil to lymphocyte ratio (NLR) as a prognostic indicator for ischemic stroke; however, some limitations remain.

Methods: We derived data from the Third China National Stroke Registry. The NLR is defined as neutrophil count/lymphocyte count. Patients included in the study were classified into four groups by NLR quartiles. Odds ratios (ORs) of adverse clinical outcomes were calculated with the lowest quartile group as the reference category. We plotted receiver operating characteristic (ROC) curves of NLR for adverse clinical outcomes and calculated area under the curve (AUC) values and cutoff values. Under different TOAST classifications, medians of NLR and ORs of adverse clinical outcomes were also calculated. Furthermore, interaction tests between NLR and etiology were performed.

Results: A total of 13,018 patients were enrolled. At both 3- and 12-month follow-ups, higher quartile groups were associated with increased risks of death and poor functional outcomes, even after adjustments. For death, the cutoff values of NLR were 3.872 at 3-month follow-up and 3.180 at 12 -month followup. For poor functional outcomes, the cutoff value of NLR was 2.846 at both 3-and 12-month followups. The association between NLR and stroke recurrence was significant only at 3-month follow-up before adjustments. There was no correlation between NLR and hemorrhagic transformation during hospitalization. Under different TOAST classifications, the medians of NLR were different; conversely, the correlations of NLR with adverse clinical outcomes had no differences.

Conclusions: High level of NLR within the first $24 \mathrm{~h}$ after admission was associated with increased risks of both short- and long-term adverse clinical outcomes in patients with ischemic stroke, regardless of etiology.

Keywords: Neutrophil to lymphocyte ratio (NLR); outcome; ischemic stroke; etiology

Submitted Feb 11, 2021. Accepted for publication May 11, 2021.

doi: $10.21037 / \mathrm{atm}-21-710$

View this article at: https://dx.doi.org/10.21037/atm-21-710 


\section{Introduction}

Ischemic stroke triggers a robust inflammatory response, resulting in brain injury development and neurological function exacerbation (1-3). However, the post-ischemic inflammatory response is a complicated process involving the induction of multiple inflammatory cell activation $(2,3)$. In the inflammatory process, ischemic tissues release chemokines and cytokines and recruit peripheral circulating leukocytes (4). Among the leukocytes infiltrating into the ischemic regions, neutrophils have been considered to play an important role in brain injury (5). Neutrophils can be recruited rapidly, within hours of ischemic stroke, to cerebral vessels (6). Many studies have shown that the infiltration of neutrophils was associated with larger infarct volumes and deterioration of ischemic brain injury $(5,7)$. Lymphocytes can also infiltrate ischemic regions during the inflammatory response, albeit several days after acute ischemic stroke, which is a later event than that of neutrophils $(4,8)$. Currently, the role of lymphocytes in ischemic brain injury is still controversial (4). Some studies have already demonstrated that lymphocytes could repair the injury via inflammation (9). At the same time, some studies have shown that lymphocytes could cause the release of proinflammatory cytokines and cytotoxic substances, which cause damage to the ischemic brain $(8,10)$. More experimental evidence has indicated that some specific lymphocyte subtypes were important cerebroprotective immunomodulators after acute ischemic stroke (11-13). Thus, neutrophils and lymphocytes might mediate differential inflammatory responses after ischemic stroke and have different effects on patients' clinical outcomes.

The neutrophil to lymphocyte ratio (NLR) is defined as neutrophil count/lymphocyte count. The NLR integrates two subtypes of leukocytes and can simultaneously reflect the balance between neutrophil and lymphocyte levels and their different immune activities. Several studies have used NLR as a prognostic indicator for ischemic stroke and found that increased NLR was associated with earlyonset delirium, mortality, or poor functional outcomes defined as modified Rankin Scale (mRS) $\geq 3$ of patients with ischemic stroke (14-19). However, generally, the sample sizes of these studies have been small, follow-up times were short, and the outcome of stroke recurrence or hemorrhagic transformation was minimally reported. Thus, we conducted this study to verify whether NLR was associated with adverse clinical outcomes defined as hemorrhagic transformation during hospitalization and recurrent stroke, all-cause death, poor functional outcomes at 3- and 12-month follow-up in ischemic stroke patients. We present the following article in accordance with the STROBE reporting checklist (available at https://dx.doi. org/10.21037/atm-21-710).

\section{Methods}

\section{Study design and population}

We derived data from the Third China National Stroke Registry (CNSR-III). The detailed design and major results of the CNSR-III have been described previously (20). Briefly, the CNSR-III is a large-scale nationwide, multicenter, prospective clinical registry study between August 2015 and March 2018 in China. A total of 15,166 patients were recruited consecutively from 201 hospitals who met the following criteria: (I) age older than 18 years; (II) diagnosis of ischemic stroke or transient ischemic attack (TIA); (III) within 7 days from the onset of symptoms to enrolment; and (IV) informed consent was provided by the patient or legally authorized representative. Acute ischemic stroke was diagnosed according to the World Health Organization (WHO) criteria and confirmed by magnetic resonance imaging (MRI) or brain computed tomography (CT). The study was conducted in accordance with the Declaration of Helsinki (as revised in 2013). The protocol and data collection of the CNSR-III were approved by the ethics committee of Beijing Tiantan Hospital (IRB approval number: KY2015-001-01) and all other participating hospitals. All patients or their legal representatives provided written informed consent before being entered into the study.

Among the enrolled patients in the CNSR-III, 14,146 were diagnosed with ischemic stroke, and therein 13,018 patients were involved in our analysis after excluding those with infection within 2 weeks before admission $(n=422)$, missing available complete blood count on admission $(\mathrm{n}=305)$, and loss to follow-up $(\mathrm{n}=401)$ (Figure 1).

\section{Baseline data collection and etiology classification}

Baseline data, including age, gender, body mass index (BMI), smoking and drinking status, medical history, National Institutes of Health Stroke Scale (NIHSS) score at admission, and time from symptom onset to enrollment and therapy were systematically collected by trained neurologists through face-to-face interviews in 


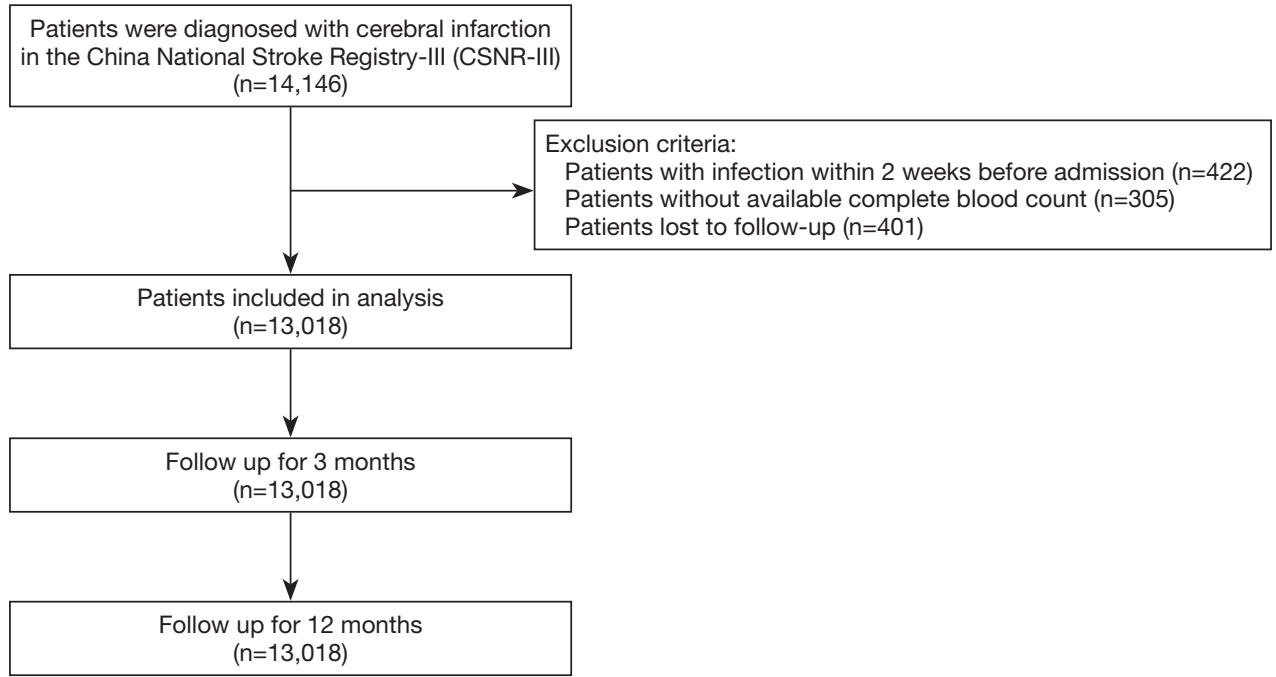

Figure 1 Flow chart.

each participating hospital. Moreover, the etiology of each patient was evaluated using Trial of Org 10172 in Acute Stroke Treatment (TOAST) classification according to clinical manifestations, imaging, and other diagnostic tests by trained neurologists at the discharge visit.

\section{Neutrophil, lymphocyte count testing, and NLR calculation}

Fasting whole blood samples from venipuncture were taken into vacutainer tubes containing ethylenediamine tetraacetic acid (EDTA) within the first $24 \mathrm{~h}$ after admission and kept at room temperature. Afterward, neutrophil and lymphocyte counts were analyzed by automated hematology analyzers at each participating hospital. All measurements were performed by laboratory personnel blinded to patients' clinical situations. The NLRs for all participants were calculated using the below formula.

NLR = neutrophil count/lymphocyte count

\section{Outcome assessment}

Hemorrhagic transformation was defined as any nontraumatic extravascular hemorrhage within a known infarct area, and imaging evidence (CT or MRI) was required, regardless of whether it caused a change in symptoms during hospitalization. Neuroradiologists reviewed the CT or MRI images with extensive experience in stroke who were blinded to patients' clinical situations.

Participants were interviewed face-to-face at 3 months and contacted over the telephone at 12 months by trained research coordinators according to the protocol of the CNSR-III. Information including functional status and cerebrovascular events were collected at each follow-up. Any incidents of all-cause death and stroke recurrence during the follow-up periods were recorded. Each case fatality was confirmed on a death certificate from the attended hospital or the local citizen registry. Recurrent stroke included both ischemic and hemorrhagic stroke, which was confirmed from the treating hospital, and suspected events without hospitalization were judged by independent endpoint judgment committees. The mRS was used to assess participants' functional dependence, and poor functional outcomes were defined as $3 \leq \mathrm{mRS} \leq 5$.

\section{Statistical analysis}

Continuous variables were presented as medians with interquartile ranges (IQR) and categorical variables as proportions. Participants were classified into four groups by NLR quartiles. The NLR ranges of quartile groups were NLR $<1.848,1.848 \leq$ NLR $<2.548,2.548 \leq$ NLR $<3.728$, and NLR $\geq 3.728$, respectively. Baseline data were compared across NLR quartile groups using the Fisher's exact test for categorical variables and the Kruskal-Wallis test for continuous variables. Firstly, both crude and adjusted odds ratios (OR) and $95 \%$ confidence intervals $(\mathrm{CI})$ of adverse clinical outcomes were calculated using the multivariate logistic regression model with the lowest NLR quartile 
group as the reference category in order to investigate the correlations between NLR and adverse clinical outcomes. Then, only adjusted ORs and $95 \%$ CIs of adverse clinical outcomes under different TOAST classifications were calculated in the same way. The interaction tests between NLR and etiology were performed to explore the difference in the relationships between NLR and adverse clinical outcomes under different etiology. To adjust for other potential confounding variables, multivariable analyses including age, gender, BMI, drinking, smoking, hypertension, lipid metabolism disorders, diabetes, previous stroke, atrial fibrillation, heart failure, heart valve disease, peripheral arterial disease, intravenous thrombolysis, endovascular therapy, time from symptom onset to enrollment, and the NIHSS score at admission were performed.

Meanwhile, participants were also grouped according to their TOAST classification, and the median with IQR of NLR in each group was calculated. The KruskalWallis test was used to compare the distribution of NLR under different etiology. Furthermore, we plotted receiver operating characteristic (ROC) curves of NLR for adverse clinical outcomes and calculated area under the curve (AUC) values and cutoff values. A $\mathrm{P}$ value $<0.05$ for two-sided hypothesis testing was considered statistically significant. All statistical analyses were performed with SAS software version 9.4 (SAS Institute Inc., Cary, NC, USA).

\section{Results}

\section{Baseline characteristics}

A total of 13,018 patients were included in our analysis. Of all participants, the median [IQR] age was 63 [54-70] years, and 4,066 (31.23\%) were female. The baseline characteristics of the participants stratified according to NLR are shown in Table 1. The medians (IQR) NLR of quartile groups were 1.500 (1.273-1.682), 2.179 (2.0162.358), 3.021 (2.784-3.338), and 5.178 (4.274-7.171), respectively. From the lowest to the highest quartile groups, the medians (IQR) NIHSS scores at admission were 3 [1-5], 3 [1-5], 3 [2-6], and 4 [2-8], and the medians (IQR) times from symptom onset to enrollment were 2 [1-4], 2 [1-4], 2 [1-4], and 2 [1-3], respectively. Compared with participants with lower NLR, those in higher NLR quartile groups were more likely to be older, male, with lower BMI, shorter time to enrollment, and higher severity of the stroke, and treated with intravenous thrombolysis or endovascular therapy.
Moreover, there were differences in smoking, drinking, hypertension, and diabetes history among participants in the NLR quartile groups. Previous stroke, atrial fibrillation, and heart failure were more frequently found in patients with higher NLR, and the other medical histories were comparable in NLR quartile groups (Table 1).

\section{Association of NLR with adverse clinical outcomes}

A total of 178 (1.37\%) participants experienced hemorrhagic transformation during hospitalization. At 3-month follow-up, 195 (1.50\%) participants had died, $833(6.40 \%)$ had experienced recurrent stroke, and 1,865 $(14.33 \%)$ were showing poor functional outcomes. While at 12 -month follow-up, $423(3.25 \%)$ participants had died, the recurrent stroke had occurred in 1,286 (9.88\%), and there were $1,778(13.66 \%)$ participants with poor functional outcomes. The risks of adverse clinical outcomes in NLR quartile groups are shown in Table 2. There was no significant correlation between NLR and hemorrhagic transformation during hospitalization. Both at 3 - and 12-month follow-ups, compared with the lowest NLR quartile group taken as reference, higher quartile groups were related to elevated risks of death and poor functional outcomes. The above associations were still significant after adjustments. At 3-month follow up compared to the lowest quartile group of NLR, the other quartile groups had 0.951 (95\% CI: 0.765 to 1.183 ), 1.147 (95\% CI: 0.930 to 1.414 ), and 1.764 (95\% CI: 1.453 to 2.142 )-fold increased risks of stroke recurrence, respectively $(\mathrm{P}=0.0479)$. However, after adjustments, the association between NLR and stroke recurrence was no longer statistically significant. The NLR was not significantly associated with recurrent stroke at 12-month follow-up (Table 2).

The ROC curves of NLR for adverse clinical outcomes are shown in Figure 2, and the corresponding AUC values and cutoff values are shown in Table 3.

\section{$N L R$ and association of NLR with adverse clinical outcomes under different etiology}

The median (IQR) of NLR in all participants was 2.548 (1.848-3.729). Under different TOAST classifications, the medians (IQR) of NLR were: 2.747 (1.972-3.981) in large artery atherosclerosis, $2.704(1.970-4.058)$ in cardiogenic embolism, 2.391 (1.770-3.295) in small artery occlusion, 2.345 (1.889-3.557) in stroke of other determined cause, and $2.520(1.818-3.750)$ in stroke of undetermined cause, 
Table 1 Baseline characteristics of the patients stratified by NLR

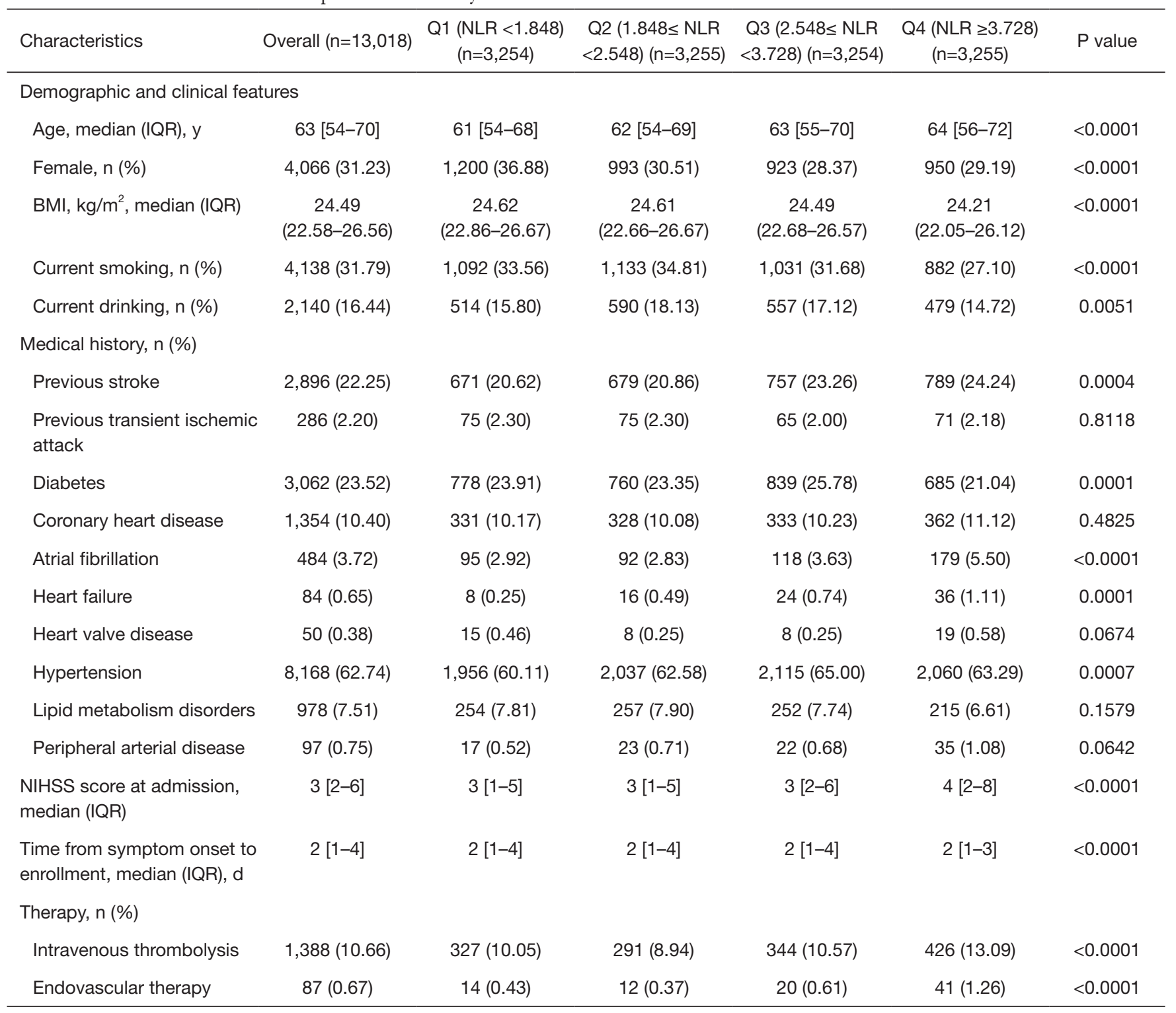

NLR, neutrophil to lymphocyte ratio; BMI, body mass index; NIHSS, National Institutes of Health Stroke Scale; IQR, interquartile range.

respectively $(\mathrm{P}<0.0001)$. Thus, NLRs were significantly different under different etiology, among which NLR was higher in patients with large artery atherosclerosis and cardiogenic embolism. The relationships between NLR and adverse clinical outcomes under different TOAST classifications are shown in Table 4. In participants with large artery atherosclerosis, NLR correlated with death and poor functional outcomes at both 3- and 12-month followups. In participants with small artery occlusion, NLR was associated with stroke recurrence at 3-month follow-up and poor functional outcomes at both 3- and 12-month follow-ups. In participants with stroke of other determined cause, NLR was related to poor functional outcomes at 12-month follow-up. A similar correlation was also found between NLR and death at 3-month follow-up in patients with stroke of undetermined cause. However, regardless of adverse clinical outcomes, the interaction $\mathrm{P}$ values of NLR and etiology were all $>0.05$. Therefore, it could be considered that there was no difference in the correlations of NLR with adverse clinical outcomes under different 
Table 2 Risks of adverse clinical outcomes stratified by NLR

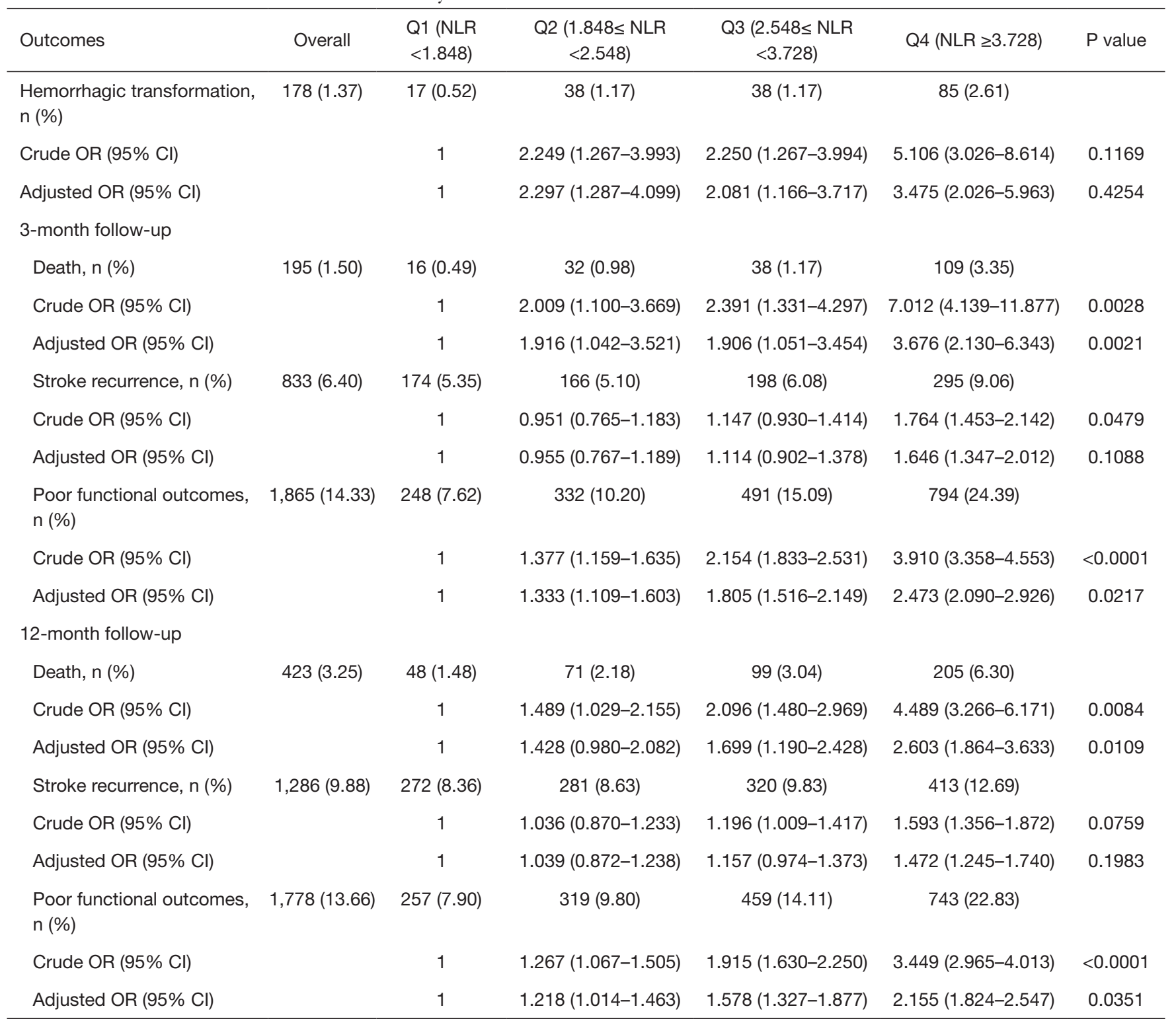

Adjusted for age, gender, BMI, drinking, smoking, hypertension, lipid metabolism disorders, diabetes, previous stroke, atrial fibrillation, heart failure, heart valve disease, peripheral arterial disease, intravenous thrombolysis, endovascular therapy, time from symptom onset to enrollment, and the NIHSS score at admission. NLR, neutrophil to lymphocyte ratio; OR, odds ratio; Cl, confidence interval; BMI, body mass index.

etiology (Table 4).

\section{Discussion}

In this analysis of the CNSR-III, we demonstrated that among participants with ischemic stroke, a high level of NLR within the first $24 \mathrm{~h}$ after admission was correlated with increased risks of both death and poor functional outcomes at 3-and 12-month follow-ups. Moreover, there might also be an association between NLR and stroke recurrence at 3-month follow-up. Although NLR was not related to hemorrhagic transformation during hospitalization, it was still considered a useful predictor for adverse clinical outcomes regardless of etiology in ischemic 

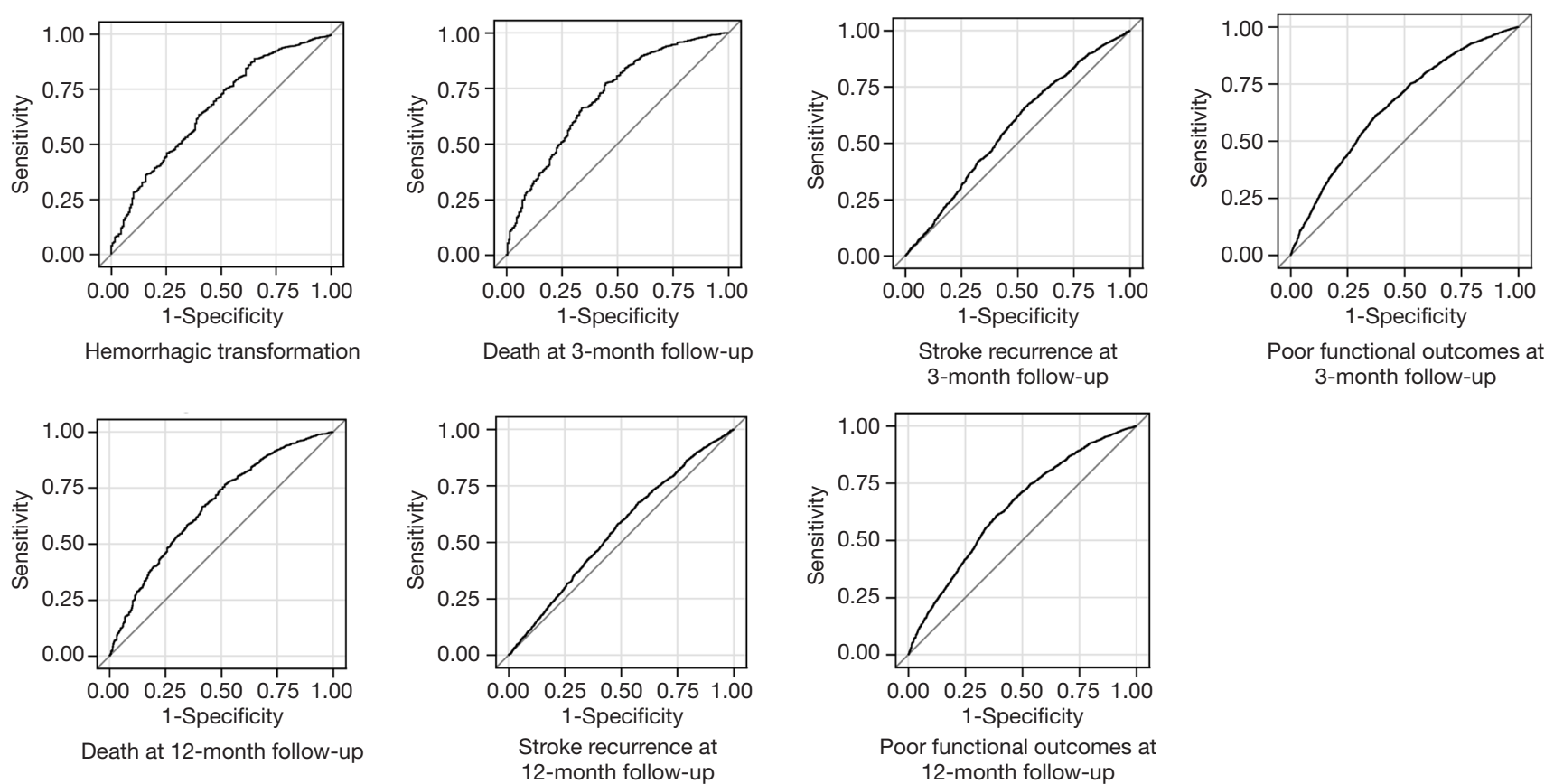

Figure 2 ROC curves of NLR for adverse clinical outcomes. ROC, receiver operating characteristic; NLR, neutrophil to lymphocyte ratio.

Table 3 Predictive values of NLR for adverse clinical outcomes

\begin{tabular}{|c|c|c|c|c|}
\hline Outcomes & $\operatorname{AUC}(95 \% \mathrm{Cl})$ & NLR cutoff & Sensitivity (\%) & Specificity (\%) \\
\hline Hemorrhagic transformation & $0.6624(0.6211-0.7036)$ & 5.371 & 34.83 & 88.86 \\
\hline \multicolumn{5}{|l|}{ 3-month follow-up } \\
\hline Death & $0.7112(0.6727-0.7496)$ & 3.872 & 55.39 & 77.31 \\
\hline Stroke recurrence & $0.5689(0.5478-0.5899)$ & 3.156 & 46.58 & 66.19 \\
\hline \multicolumn{5}{|l|}{ 12-month follow-up } \\
\hline Death & $0.6667(0.6396-0.6938)$ & 3.180 & 58.39 & 66.69 \\
\hline Stroke recurrence & $0.5546(0.5376-0.5716)$ & 3.213 & 42.54 & 67.48 \\
\hline Poor functional outcomes & $0.6423(0.6283-0.6564)$ & 2.846 & 61.31 & 60.92 \\
\hline
\end{tabular}

NLR, neutrophil to lymphocyte ratio; AUC, area under the curve; $\mathrm{Cl}$, confidence interval.

stroke patients in either the short- or long-term.

Similar to our study, most recent studies, which investigated the relationship between NLR and prognosis of ischemic stroke patients, used the level of NLR within the first $24 \mathrm{~h}$ after admission. It has been indicated that the NLR calculated at 48-72 h after acute ischemic stroke was identified as the strongest predictor of patients' outcomes (21). The median (IQR) time from symptom onset to enrollment in our study was 2 [1-4] days, and the blood samples were taken within the first $24 \mathrm{~h}$ after admission. Thus, the levels of NLR calculated in our study had a stronger predictive value for clinical outcomes in ischemic stroke patients.

Several recent studies have found that NLR was an independent predictor of 3-month mortality in acute ischemic stroke patients $(14,15)$. A meta-analysis that involved 9 studies in 2017 defined poor functional outcomes of ischemic stroke as $\mathrm{mRS} \geq 3$ and indicated that for patients 
Table 4 Risks of adverse clinical outcomes stratified by NLR under different TOAST classification

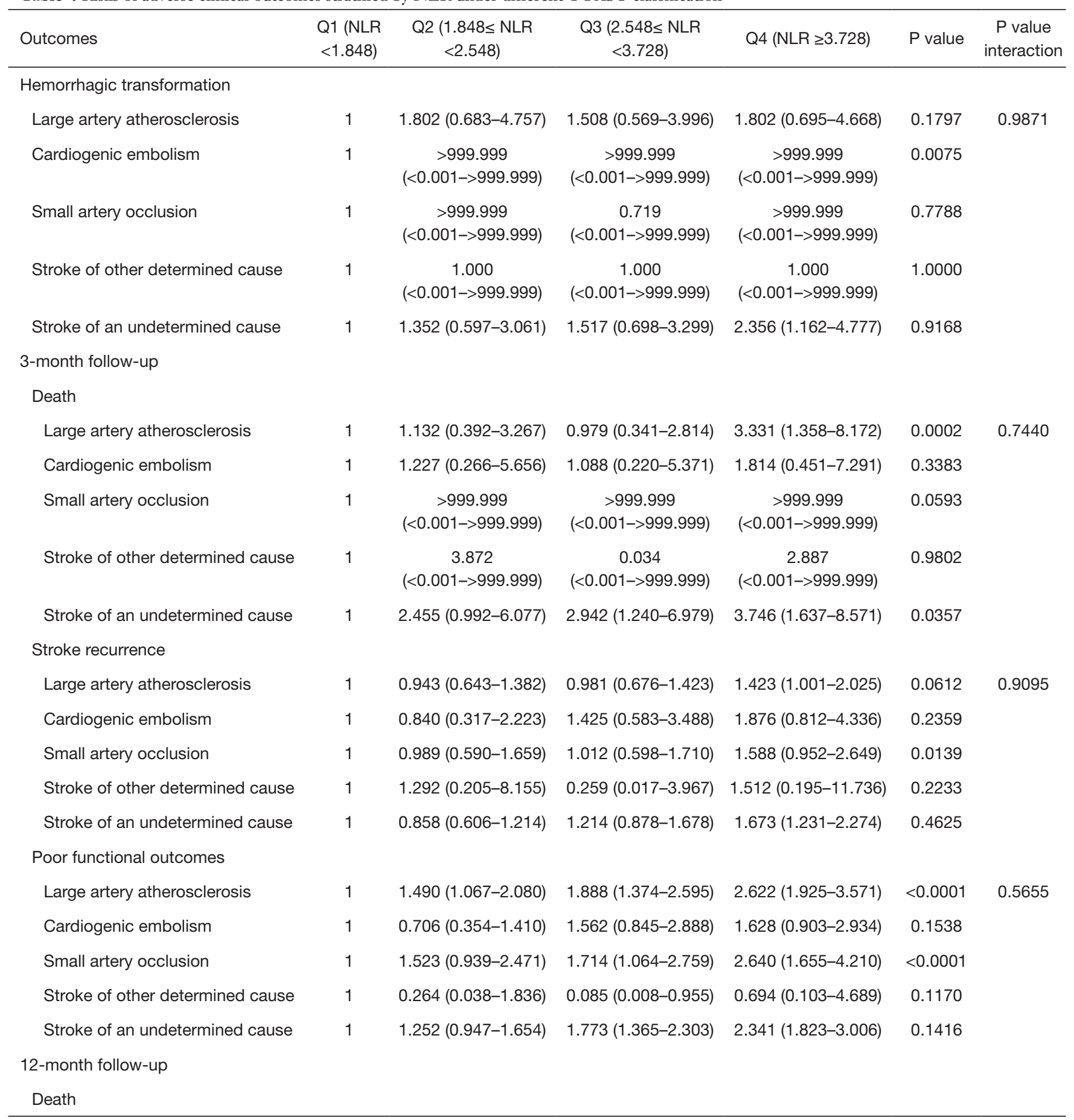

Table 4 (continued) 
Table 4 (continued)

\begin{tabular}{|c|c|c|c|c|c|c|}
\hline Outcomes & $\begin{array}{l}\text { Q1 (NLR } \\
<1.848)\end{array}$ & $\begin{array}{c}\text { Q2 }(1.848 \leq \text { NLR } \\
<2.548)\end{array}$ & $\begin{array}{c}\text { Q3 }(2.548 \leq \mathrm{NLR} \\
<3.728)\end{array}$ & Q4 (NLR $\geq 3.728)$ & $P$ value & $\begin{array}{c}\mathrm{P} \text { value } \\
\text { interaction }\end{array}$ \\
\hline Large artery atherosclerosis & 1 & $1.360(0.669-2.767)$ & $1.499(0.763-2.943)$ & 2.719 (1.449-5.102) & 0.0015 & 0.7121 \\
\hline Small artery occlusion & 1 & $1.328(0.392-4.505)$ & $1.361(0.412-4.494)$ & $2.624(0.858-8.023)$ & 0.2607 & \\
\hline Stroke of an undetermined cause & 1 & $1.547(0.871-2.749)$ & $2.333(1.378-3.951)$ & $2.714(1.636-4.501)$ & 0.0713 & \\
\hline \multicolumn{7}{|l|}{ Stroke recurrence } \\
\hline Large artery atherosclerosis & 1 & $1.164(0.841-1.612)$ & $1.201(0.876-1.649)$ & $1.395(1.021-1.905)$ & 0.1279 & 0.7863 \\
\hline Cardiogenic embolism & 1 & $0.612(0.285-1.317)$ & $1.449(0.745-2.817)$ & $1.328(0.700-2.522)$ & 0.1386 & \\
\hline Stroke of an undetermined cause & 1 & $0.973(0.742-1.277)$ & $1.140(0.873-1.488)$ & $1.631(1.268-2.098)$ & 0.5626 & \\
\hline \multicolumn{7}{|l|}{ Poor functional outcomes } \\
\hline Large artery atherosclerosis & 1 & $1.527(1.091-2.138)$ & $1.747(1.267-2.410)$ & $2.183(1.594-2.988)$ & 0.0007 & 0.8725 \\
\hline Cardiogenic embolism & 1 & $1.107(0.574-2.134)$ & $1.358(0.724-2.548)$ & $2.042(1.144-3.643)$ & 0.1085 & \\
\hline Small artery occlusion & 1 & $1.299(0.794-2.125)$ & $1.508(0.934-2.437)$ & $2.153(1.339-3.461)$ & 0.0045 & \\
\hline Stroke of other determined cause & 1 & $1.838(0.263-12.863)$ & $0.829(0.097-7.096)$ & $3.179(0.408-24.748)$ & 0.0208 & \\
\hline Stroke of an undetermined cause & 1 & $0.983(0.747-1.295)$ & $1.491(1.157-1.923)$ & $2.017(1.584-2.567)$ & 0.1984 & \\
\hline
\end{tabular}

Adjusted for age, gender, BMI, drinking, smoking, hypertension, lipid metabolism disorders, diabetes, previous stroke, atrial fibrillation, heart failure, heart valve disease, peripheral arterial disease, intravenous thrombolysis, endovascular therapy, time from symptom onset to enrollment and the NIHSS score at admission. NLR, neutrophil to lymphocyte ratio; TOAST, Trial of Org 10172 in Acute Stroke Treatment; BMI, body mass index.

with ischemic stroke, higher NLR was correlated with poorer functional outcomes at 3-month follow-up (10). Our study came to a similar conclusion, which was that a high level of NLR was associated with increased risks of both short-term death and poor functional outcomes. The corresponding cutoff values of NLR were 3.872 (sensitivity $55.39 \%$, specificity $77.31 \%$ ) for death and 2.846 (sensitivity $62.57 \%$, specificity $61.30 \%$ ) for poor functional outcomes, respectively. Moreover, we extended the follow-up time and found the above association remained significant at 12 -month follow-up. NLR $\geq 3.180$ (sensitivity 58.39\%, specificity $66.69 \%$ ) could predict death and NLR $\geq 2.846$ (sensitivity $61.31 \%$, specificity $60.92 \%$ ) could predict poor functional outcomes. Thus, NLR could be regarded as a predictor for both short- and long-term adverse clinical outcomes in ischemic stroke patients. However, little is known about the immunological mechanisms underlying the above association.

After ischemic stroke, neutrophils migrate immediately, even against blood flow, then transmigrate out of blood vessels and become the first immune cells to invade the injured brain tissue (22). Additionally, the local blood-brain barrier breakdown caused by ischemia promotes neutrophil entry (23). At 48-72 h after ischemic stroke, the neutrophil invasion reaches the zenith, and neutrophils aggravate ischemic injury through a variety of different actions (24). On the one hand, neutrophil aggregation in cerebral vessels might block microvessels, impede perfusion, and eventually lead to prolonged ischemia (6). On the other hand, neutrophils could induce further thrombus formation by releasing molecules to participate in neutrophil extracellular trap formation or interacting with platelets directly (25). 
Besides, neutrophils also lead to the release of deleterious substances or inflammatory mediators, thus potentiating ischemic damage (25). In experimental brain ischemia models, various therapeutic interventions, by blocking proinflammatory cytokines to inhibit neutrophil infiltration or blocking the neutrophil pro-inflammatory function, successfully reduced the infarct volume and improved neurological outcomes $(25,26)$.

Lymphocytes also accumulate in the post-ischemic brain but more slowly than neutrophils. Different subtypes of lymphocytes exert different effects on cerebral ischemia $(8,27)$. Pro-inflammatory lymphocytes, such as T helper type 1 (TH1) and T helper type 17 (TH17), have been thought to worsen stroke outcomes (28-30). In contrast, regulatory $\mathrm{T}$ cells (Treg) have been characterized as diseaselimiting protective cells, and their percentage within the $\mathrm{T}$ helper cell population is increased in brains after stroke (31). It is acknowledged that Tregs can modulate various immunologic pathways and maintain immune homeostasis by interacting with other cells directly and producing antiinflammatory cytokines (32). In an experimental stroke model, the selective depletion of Tregs had a link with an increase in infarct volume and behavioral deficits after brain ischemia (11). Besides, regulatory B cells also showed the capacity to limit central nervous system inflammation and neurological deficits in murine experimental stroke (13).

In summary, neutrophils and lymphocytes affect acute brain ischemic injury through different actions, and their functional role might be much more complicated and diversified than currently appreciated. As an inflammatory marker that integrates both neutrophils and lymphocytes, the NLR could reflect the imbalance of post-stroke immunization more accurately than leukocyte or neutrophil counts. Moreover, NLR is much easier to evaluate than many other indicators.

Currently, limited studies are investigating the relationship between NLR and stroke recurrence in ischemic stroke patients. It has previously been indicated that high levels of neutrophil ratio were associated with an increased risk of new stroke in patients with a minor ischemic stroke or TIA (33). Our study showed there might also be a correlation between the level of NLR above 3.156 (sensitivity $46.58 \%$, specificity $66.19 \%$ ) and elevated risks of stroke recurrence at 3-month follow-up; however, further research is required to validate this finding.

Studies by Maestrini and Guo demonstrated that NLR was independently correlated with symptomatic intracranial hemorrhage after intravenous thrombolysis in patients with ischemic stroke $(34,35)$. At the same time, Goyal and Duan also reported that higher NLR was an independent predictor of symptomatic intracranial hemorrhage after endovascular treatment in patients with ischemic stroke (36,37). A recent meta-analysis involving 7 studies showed that an NLR with a cutoff value of 7.5-11 could predict hemorrhagic transformation in acute ischemic stroke patients (38). However, we found that NLR was not related to ischemic stroke patients' hemorrhagic transformation during hospitalization. The studies mentioned above were based on the observation of ischemic stroke patients under reperfusion therapy whose risks of hemorrhagic transformation were higher, which we thought might explain the difference between our study and those mentioned above.

Our study detected differences in NLR under different etiology. In particular, among patients with large artery atherosclerosis and cardiogenic embolism, NLR was significantly higher. This might be because post-ischemic inflammatory responses are different in patients with different TOAST classifications. Atherosclerosis is a chronic inflammatory process. In patients with large artery atherosclerosis, ischemic stroke might trigger a much stronger inflammatory response based on this chronic inflammatory process. In patients with cardiogenic embolism, the neurological deficit always peaks rapidly, and the infarct volume is usually large, causing a strong, rapid inflammatory response. These factors might explain the higher NLR in patients with large artery atherosclerosis and cardiogenic embolism. Our results were consistent with those of most previous studies; however, NLR has also been reported to not vary significantly among the stroke subtypes (39). Furthermore, our study indicated that the relationships between NLR and adverse clinical outcomes did not differ between patients with different TOAST classifications, which suggested that the different post-ischemic inflammatory responses might have similar impacts on the clinical outcomes of patients with different etiology. However, more recently, studies focused on NLR associations with adverse clinical outcomes in patients with ischemic stroke under different etiology have been limited, and our results need to be confirmed through future research.

There were several limitations to our study. Firstly, heterogeneity of equipment in participating hospitals may have led to biased estimates of association, but this might have had little impact on results due to daily practice and strict quality control in every participating hospital. 
Secondly, our study only collected the level of NLR at baseline and did not have dynamic data about NLR or evaluate the change of NLR during hospitalization. However, the dynamic change of NLR over time might provide valuable information to understand the underlying mechanism of inflammatory response after ischemic stroke. Thirdly, the median (IQR) of the NIHSS score at the admission of all participants in our study was 3 [2-6]. The severity of ischemic stroke may have been mild, and this could have limited the ability to estimate the association of NLR with clinical outcomes in more severe ischemic stroke patients. Finally, residual bias might have remained because of the influence of comorbidities or environmental factors such as tumor, trauma, and acute toxicosis.

\section{Conclusions}

The NLR within the first $24 \mathrm{~h}$ after admission was significantly higher in patients with large artery atherosclerosis and cardiogenic embolism. Regardless of etiology, a high level of NLR was associated with increased risks of both short- and long-term adverse clinical outcomes in patients with ischemic stroke.

\section{Acknowledgments}

We extend our thanks to all study participants, their relatives, the survey team members at participating hospitals of the CNSR-III, and the project development and management teams at the Beijing Tiantan Hospital.

Funding: This study was supported by grants from National Key R\&D Program of China (2018YFC1312903), grants from Beijing Municipal Science \& Technology Commission (D171100003017002, Z181100001818001), and grants from National Science and Technology Major Project (2017ZX09304018).

\section{Footnote}

Reporting Checklist: The authors have completed the STROBE reporting checklist. Available at https://dx.doi. org/10.21037/atm-21-710

Peer Review File: Available at https://dx.doi.org/10.21037/ atm-21-710

Conflicts of Interest: All authors have completed the ICMJE uniform disclosure form (available at https://dx.doi. org/10.21037/atm-21-710). All authors report that this study was supported by grants from National Key R\&D Program of China (2018YFC1312903), grants from Beijing Municipal Science \& Technology Commission (D171100003017002, Z181100001818001), and grants from National Science and Technology Major Project (2017ZX09304018)

Ethical Statement: The authors are accountable for all aspects of the work in ensuring that questions related to the accuracy or integrity of any part of the work are appropriately investigated and resolved. The study was conducted in accordance with the Declaration of Helsinki (as revised in 2013). The ethics committee at Beijing Tiantan Hospital (IRB approval number: KY2015-001-01) and all study centers gave ethical approval of the CNSR-III study protocol. Written consent was provided by all participants or their legal representatives.

Open Access Statement: This is an Open Access article distributed in accordance with the Creative Commons Attribution-NonCommercial-NoDerivs 4.0 International License (CC BY-NC-ND 4.0), which permits the noncommercial replication and distribution of the article with the strict proviso that no changes or edits are made and the original work is properly cited (including links to both the formal publication through the relevant DOI and the license). See: https://creativecommons.org/licenses/by-nc-nd/4.0/.

\section{References}

1. Chamorro A, Hallenbeck J. The harms and benefits of inflammatory and immune responses in vascular disease. Stroke 2006;37:291-3.

2. Macrez R, Ali C, Toutirais O, et al. Stroke and the immune system: from pathophysiology to new therapeutic strategies. Lancet Neurol 2011;10:471-80.

3. Chamorro Á, Meisel A, Planas AM, et al. The immunology of acute stroke. Nat Rev Neurol 2012;8:401-10.

4. Kim JY, Park J, Chang JY, et al. Inflammation after ischemic stroke: the role of leukocytes and glial cells. Exp Neurobiol 2016;25:241-51.

5. Herz J, Sabellek P, Lane TE, et al. Role of neutrophils in exacerbation of brain injury after focal cerebral ischemia in hyperlipidemic mice. Stroke 2015;46:2916-25.

6. Kleinig TJ, Vink R. Suppression of inflammation in ischemic and hemorrhagic stroke: therapeutic options. Curr Opin Neurol 2009;22:294-301. 
7. Buck BH, Liebeskind DS, Saver JL, et al. Early neutrophilia is associated with volume of ischemic tissue in acute stroke. Stroke 2008;39:355-60.

8. Kim JY, Kawabori M, Yenari MA. Innate inflammatory responses in stroke: mechanisms and potential therapeutic targets. Curr Med Chem 2014;21:2076-97.

9. Xue J, Huang W, Chen X, et al. Neutrophil-to-lymphocyte ratio is a prognostic marker in acute ischemic stroke. J Stroke Cerebrovasc Dis 2017;26:650-7.

10. Zhang J, Ren Q, Song Y, et al. Prognostic role of neutrophil-lymphocyte ratio in patients with acute ischemic stroke. Medicine (Baltimore) 2017;96:e8624.

11. Liesz A, Suri-Payer E, Veltkamp C, et al. Regulatory T cells are key cerebroprotective immunomodulators in acute experimental stroke. Nat Med 2009;15:192-9.

12. Liesz A, Zhou W, Na SY, et al. Boosting regulatory Tcells limits neuroinflammation in permanent cortical stroke. J Neurosci 2013;33:17350-62.

13. Ren X, Akiyoshi K, Dziennis S, et al.Regulatory B cells limit CNS inflammation and neurologic deficits in murine experimental stroke. J Neurosci 2011;31:8556-63.

14. Tokgoz S, Keskin S, Kayrak M, et al. Is neutrophil/ lymphocyte ratio predict to short-term mortality in acute cerebral infarct independently from infarct volume?. J Stroke Cerebrovasc Dis 2014;23:2163-8.

15. Kocaturk O, Besli F, Gungoren F, et al. The relationship among neutrophil to lymphocyte ratio, stroke territory, and 3-month mortality in patients with acute ischemic stroke. Neurol Sci 2019;40:139-46.

16. Yu S, Arima H, Bertmar C, et al. Neutrophil to lymphocyte ratio and early clinical outcomes in patients with acute ischemic stroke. J Neurol Sci 2018;387:115-8.

17. Qun S, Tang Y, Sun J, et al. Neutrophil-to-lymphocyte ratio predicts 3-month outcome of acute ischemic stroke. Neurotox Res 2017;31:444-452.

18. Celikbilek A, Ismailogullari S, Zararsiz G. Neutrophil to lymphocyte ratio predicts poor prognosis in ischemic cerebrovascular disease. J Clin Lab Anal 2014;28:27-31.

19. Kotfis K, Bott-Olejnik M, Szyli ska A, et al. Could Neutrophil-to-Lymphocyte Ratio (NLR) Serve as a Potential Marker for Delirium Prediction in Patients with Acute Ischemic Stroke? A Prospective Observational Study. J Clin Med 2019;8:1075.

20. Wang Y, Jing J, Meng X, et al. The third china national stroke registry (CNSR-III) for patients with acute ischaemic stroke or transient ischaemic attack: design, rationale and baseline patient characteristics. Stroke Vasc Neurol 2019;4:158-64.
21. Petrone AB, Eisenman RD, Steele KN, et al. Temporal dynamics of peripheral neutrophil and lymphocytes following acute ischemic stroke. Neurol Sci 2019;40:1877-85.

22. Neumann J, Riek-Burchardt M, Herz J, et al. Very-lateantigen-4 (VLA-4)-mediated brain invasion by neutrophils leads to interactions with microglia, increased ischemic injury and impaired behavior in experimental stroke. Acta Neuropathol 2015;129:259-77.

23. Tang G, Liu Y, Zhang Z, et al. Mesenchymal stem cells maintain blood-brain barrier integrity by inhibiting aquaporin-4 upregulation after cerebral ischemia. Stem Cells 2014;32:3150-62.

24. Jickling GC, Liu D, Ander BP, et al. Targeting neutrophils in ischemic stroke: translational insights from experimental studies. J Cereb Blood Flow Metab 2015;35:888-901.

25. Ruhnau J, Schulze J, Dressel A, et al. Thrombosis, Neuroinflammation, and Poststroke Infection: The Multifaceted Role of Neutrophils in Stroke. J Immunol Res 2017;2017:5140679.

26. Wang Q, Tang XN, Yenari MA. The inflammatory response in stroke. J Neuroimmunol 2007;184:53-68.

27. Jin R, Yang G, Li G. Inflammatory mechanisms in ischemic stroke: role of inflammatory cells. J Leukoc Biol 2010;87:779-89.

28. Shichita T, Sugiyama Y, Ooboshi H, et al. Pivotal role of cerebral interleukin-17-producing gammadeltat cells in the delayed phase of ischemic brain injury. Nat Med 2009;15:946-50.

29. Gelderblom M, Weymar A, Bernreuther C, et al. Neutralization of the IL-17 axis diminishes neutrophil invasion and protects from ischemic stroke. Blood 2012;120:3793-802.

30. Liesz A, Zhou W, Mracsko E, et al. Inhibition of lymphocyte trafficking shields the brain against deleterious neuroinflammation after stroke. Brain 2011;134:704-20.

31. Liesz A, Hu X, Kleinschnitz C, et al. Functional Role of Regulatory Lymphocytes in Stroke: facts and controversies. Stroke 2015;46:1422-30.

32. Bonaventura A, Liberale L, Vecchié A, et al. Update on Inflammatory Biomarkers and Treatments in Ischemic Stroke. Int J Mol Sci 2016,17:E1967.

33. Zhu B, Pan Y, Jing J, et al. Neutrophil counts, neutrophil ratio, and new stroke in minor ischemic stroke or TIA. Neurology 2018;90:e1870-e1878.

34. Maestrini I, Strbian D, Gautier S, et al. Higher neutrophil counts before thrombolysis for cerebral ischemia predict worse outcomes. Neurology 2015;85:1408-16. 
35. Guo Z, Yu S, Xiao L, et al. Dynamic change of neutrophil to lymphocyte ratio and hemorrhagic transformation after thrombolysis in stroke. J Neuroinflammation 2016;13:199.

36. Duan Z, Wang H, Wang Z et al. Neutrophil-Lymphocyte Ratio Predicts Functional and Safety Outcomes after Endovascular Treatment for Acute Ischemic Stroke. Cerebrovasc Dis 2018;45:221-7.

37. Goyal N, Tsivgoulis G, Chang JJ, et al. Admission neutrophil-to-lymphocyte ratio as a prognostic biomarker of outcomes in large vessel occlusion strokes. Stroke

Cite this article as: Quan K, Wang A, Zhang X, Meng X, Chen P, Li H, Wang Y. Neutrophil to lymphocyte ratio and adverse clinical outcomes in patients with ischemic stroke. Ann Transl Med 2021;9(13):1047. doi: 10.21037/atm-21-710
2018;49:1985-7.

38. Zhang R, Wu X, Hu W, et al. Neutrophil-to-lymphocyte ratio predicts hemorrhagic transformation in ischemic stroke: A meta-analysis. Brain Behav 2019;9:e01382.

39. Harpaz D, Seet RCS, Marks RS, et al. Blood-Based Biomarkers Are Associated with Different Ischemic Stroke Mechanisms and Enable Rapid Classification between Cardioembolic and Atherosclerosis Etiologies. Diagnostics 2020;10:804.

(English Language Editors: J. Jones and J. Chapnick) 